\title{
Um modelo conceitual para o processo de transferência do conhecimento em cluster
}

\author{
Vânia Simões Lopes Fioravanti Mestranda em Administração. Universidade Paulista (UNIP) - Brasil. vania.simoes.lopes@gmail.com \\ Flávio Romero Macau Doutor em Estratégia de Operações. Universidade Paulista (UNIP) - Brasil. professor@flaviomacau.com
}

\section{RESUMO}

Na transferência do conhecimento entre organizações inseridas em clusters, há fatores que podem inibir ou facilitar esse processo, levando a uma melhor gestão, melhorando e facilitando o fluxo entre os participantes. O objetivo deste trabalho é apresentar um modelo conceitual para estudo do processo de transferência do conhecimento em cluster, identificando fatores que influenciam este processo. Este trabalho propõe um estudo exploratório, conceitual, a partir de um estudo bibliográfico com coleta e análise de dados secundários, decorrentes de publicações científicas, desenvolvida a partir de artigos de periódicos de 2011 a 2016 sobre transferência do conhecimento, redes e cluster. Verificou-se que a transferência do conhecimento sofre a influência de diferentes fatores, que podem impactar esse processo, na medida em que organizações inseridas em cluster têm dimensões distintas, tais como cooperação, relação com instituições de apoio, mobilidade da força de trabalho e proximidade espacial. Foram delineados quatro pressupostos e fatores que facilitam ou inibem a transferência do conhecimento entre organizações, que podem orientar os estudos sobre clusters, tendo assim uma contribuição conceitual e metodológica. Como contribuição prática gerencial, essa base pode orientar gestores em estratégias que visem explorar o potencial dos clusters.

Palavras chave: Conhecimento. Redes. Cluster. Parque Tecnológico.

\section{A conceptual model for knowledge transfer in clusters}

\begin{abstract}
The transfer of knowledge between organizations embedded in clusters can present factors that inhibit or facilitate this process, leading to better management, improving and facilitating the flow of knowledge among participants. The aim of this paper is to present a conceptual model for studies of the process of knowledge transfer in clusters, identifying factors that influence the process. This paper proposes an exploratory, conceptual study from a bibliographic study with collection and analysis of secondary data resulting from scientific publications, developed from 2011 to 2016 journal articles on transfer of knowledge, networks and cluster. It was found that the transfer of knowledge is influenced by different factors that can impact this process, to the extent that the process between organizations embedded in cluster has different dimensions (cooperation, relationship with support institutions, mobility of the workforce and spatial proximity). Four assumptions were outlined, as well as the factors that facilitate or inhibit the transfer of knowledge between organizations. These can guide studies of clusters, thus making a conceptual and methodological contribution to the field. The managerial practical contribution is the use of this model by managers to guide strategies aimed at exploring the potential of clusters.
\end{abstract}

Keywords: Knowledge. Business Network. Cluster. Science Park. 


\section{INTRODUÇÃO}

Embora o conhecimento sempre tenha sido reconhecido como relevante para o desenvolvimento da sociedade e das organizações, o tema ganhou relevância maior a partir da década de 1990, devido principalmente à aceleração do desenvolvimento tecnológico. Na medida em que os efeitos da globalização tornaram as fronteiras entre organizações menos claras (TODEVA; KNOKE; KESKINOVA, 2007), o conhecimento vindo da ciência, tecnologia e inovação se tornou indissociável do desenvolvimento dos países e empresas. A conceituação de conhecimento é algo ainda sem consenso. Um caminho está em considerar que dados brutos trabalhados se tornam informações, que ao apresentarem utilidade para uso específico tornam-se conhecimento (STAIR; REYNOLDS, 2002; DAVENPORT; PRUSAK, 1998). O conhecimento se multiplica, pois uma vez transferido permanece com o emissor e também enriquece o receptor (CASTELLS; CARDOSO, 2005). Nesse sentido, organizações atuando de forma conectada, por meio de redes, podem conjuntamente desenvolver sistemas de troca de informações e conhecimento, a partir da interação com outras organizações, com benefícios para todos os envolvidos (NONAKA; TAKEUCHI, 1997; KRAATZ, 1998; POWELL, 1998). Um fator de destaque em uma rede é justamente sua habilidade de distribuir conhecimento entre seus integrantes, pois proteger recursos e competências como ativos de propriedade exclusiva é cada vez menos possível, restando aos gestores compartilhar (SAWHNEY; PARIKH, 2001).

Do ponto de vista teórico, as redes podem ser estudadas a partir de diferentes abordagens teóricas, como os estudos sobre cluster, entendido como a concentração geográfica de empresas. O reconhecimento de que desempenho superior resultam essa concentração geográfica tem sido visto em diversos estudos (TODEVA, 2006; TODEVA; KNOKE; KESKINOVA, 2007). Para alguns autores, clusters e redes são conceitos próximos (EBERS; JARILLO, 1998), enquanto outros consideram que um cluster pode ser reconhecido como um tipo particular de rede, focada em uma concentração setorial e geográfica (AMATO NETO, 2000). As diferentes abordagens não devem ser vistas como excludentes, e sim como complementares (OLIVER; EBERS, 1998). A formação da rede ou a participação em um cluster podem não ser suficientes, pois para alcançar os objetivos desejados pelos participantes são envolvidos muitos fatores, inclusive de governança (BALESTRIN; VERSCHOORE, 2008). 


\subsection{Problema de pesquisa e objetivo}

$\mathrm{Na}$ transferência do conhecimento entre organizações há fatores que podem inibir ou facilitar esse processo, levando a uma melhor gestão, melhorando e facilitando o fluxo entre os participantes. Na atuação em um cluster essa relação de organizações pode ser caracterizada como um problema de pesquisa expressado pela pergunta: Como o cluster influencia o processo de transferência de conhecimento entre as organizações? O objetivo deste trabalho é apresentar uma proposta de modelo conceitual para estudo do processo de transferência do conhecimento entre organizações participantes de um cluster, por meio da identificação de fatores que interferem neste processo, tendo uma contribuição de natureza conceitual e metodológica.

\section{FUNDAMENTAÇÃO TEÓRICA}

O tema de atuação em redes infere a possível existência de vantagens obtidas nessa formação, particularmente no aspecto do potencial para a transferência do conhecimento. Castells e Cardoso (2005) destacam que a sociedade em rede é uma estrutura social baseada em redes que geram, processam e distribuem informação e o conhecimento. Em ambientes dinâmicos e de rápida mudança são fundamentais a utilização do conhecimento e do potencial de aprendizagem organizacional (BROWN; EISENHARDT, 1997). Assim, a atuação em redes, constituída a partir da inserção em um cluster, pode potencialmente aumentar a capacidade de transferência do conhecimento entre as organizações.

\subsection{Redes de negócios}

É aceita a noção de que a estrutura das empresas e demais tipos de organizações públicas e privadas inclui também seu conjunto de relações. Os argumentos de que a sociedade está organizada em redes de conexões estão fundamentados em autores como Castells (2000), Nohria e Ecles (1992), Granovetter (1985) e Uzzi (1997). A afirmativa convergente é de que toda empresa está em rede, quer seus atores tenham, ou não, consciência dessa situação. Nesse contexto está inserido o termo redes como uma noção abstrata que se refere a um conjunto de nós e relações que os conectam, podendo ser aplicado a indivíduos e organizações (FOMBRUN, 1982).

A perspectiva econômica nos estudos em redes busca capturar os aspectos econômicos desse fenômeno. Williamson (1979) constata que uma empresa não é apenas uma organização fabricante de produtos ou serviços, mas uma estrutura de relações da qual emergem custos de transação, oriundos da necessidade das empresas se relacionarem. A importância de atuação 
econômica conjunta é evidenciada também por Ballou, Gilbert e Mukherjee (2000) ao afirmarem que oportunidades de ganhos são potencializadas ao se explorar as relações entre empresas, no que os autores chamaram de coordenação interorganizacional. Já teóricos estratégicos enfatizam as questões de estrutura das organizações e a necessidade de coordenação entre empresas para melhor atingir seus objetivos, gerando assim uma nova forma organizacional que substitui a forma multidivisional como maneira dominante de estruturar uma empresa (MINTZBERG, 1979; MILES; SNOW, 1986).

Para Balestrin e Verschoore (2008) empresas podem atuar em conjunto em uma rede de negócios, buscando obter escala e poder de mercado, acesso a soluções conjuntas, aprendizagem e inovação, redução de custos e compartilhamento de riscos, por meio de relações sustentáveis e de longo. Assim, uma rede pode ser vista como um conjunto de nós interconectados, ou seja, indivíduos e organizações independentes atuando conjuntamente (CASTELLS, 2000). Apesar das diferentes abordagens, o ponto de convergência é a necessidade de atuação conjunta para atingir um desempenho superior. Este trabalho segue esse paradigma da sociedade em redes.

\subsection{Cluster}

A literatura apresenta diferentes formas de entender os diferentes arranjos organizacionais que tentam atender a essa necessidade e os ganhos resultantes dessas relações, usando termos como alianças, parcerias, redes, cluster ou joint ventures (GRANDORI; SODA, 1995; TODEVA, 2006). $\mathrm{Na}$ medida em que surgem novos arranjos organizacionais que não podem ser inteiramente explicados pelo corpo de teorias econômicas existentes, verifica-se o aumento de interesse no fenômeno de aglomerações geográficas de organizações (TALLMAN et al, 2004; MASCENA; FIGUEIREDO; BOAVENTURA, 2013), que pode explicar o desempenho econômico superior de determinadas regiões (ZENG et al, 2008).

As organizações que formam uma aglomeração costumam apresentar uma mistura atípica de competição e cooperação. A importância das aglomerações de empresas para economia pode ser observada no trabalho de Marshall (1890), ao investigar os distritos industriais da Inglaterra. Porter (1990) fez uso do termo cluster para se referir à concentrações geográficas de empresas de um segmento específico, de modo a aumentar sua capacidade competitiva por meio da proximidade geográfica, observado desde a Idade Média, propiciando melhor acesso a empregados, fornecedores, instituições de apoio e informações. Alguns autores fazem ainda a distinção entre cluster e Arranjo Produtivo Local (APL), tendo como principal ponto de diferenciação estar na atuação governamental. Ao passo que cluster pode ter se formado de forma espontânea, não induzida, os APLs têm envolvimento do governo, por meio de órgãos de fomento 
(FIGUEIREDO; DI SÉRIO, 2007). Enquanto o elemento principal do cluster reside na proximidade geográfica dos seus integrantes, para redes, este fator está ligado aos relacionamentos de troca. Embora não sejam manifestações recentes, redes e cluster podem ser elementos capazes de explicar o desenvolvimento diferenciado de determinada região (ZACCARELLI et al, 2008).

Para Zaccarelli et al (2008) apenas a aglomeração de empresas não caracteriza um cluster, mas somente quando no agrupamento houver interação entre as empresas participantes, gerando características competitivas. Já para Todeva (2006) clusters são regionais e constituídos por firmas interconectadas, levando a grupos setoriais formados por diferentes empresas. Segundo Lastres e Cassiolato (2003), o termo cluster refere-se a aglomerados territoriais que desenvolvem atividades similares, devendo, portanto, ser percebido como um sistema, podendo ter um nível de padrão de atividades altamente semelhantes (TODEVA, 2006). Os conceitos de teorias de redes, quando aliados aos efeitos da proximidade espacial, se tornam uma importante combinação conceitual para entender como setores desenvolvem habilidades e conhecimento mais rapidamente que outros.

\subsection{Gestão do Conhecimento}

A definição de conhecimento é discutida desde a Antiguidade, por debates filosóficos como em Platão e Aristóteles. Essa discussão ressurge na sociedade contemporânea, no contexto dos trabalhos de Polanyi (1967) sobre o conhecimento na sua dimensão tácita e explícita e seu papel nas organizações, e na discussão no âmbito das organizações sobre inovação de produtos e disseminação de tecnologia (CLARK; FUJIMOTO, 1991; ALLEN, 1977). O final da década de 1990 marca o início da utilização da Internet como ferramenta comercial, mudando o padrão das relações entre pessoas e organizações e intensificando a ideia de uma sociedade em rede, na qual o conhecimento é ainda mais partilhado. Na chamada nova economia, conhecimento - não mais o maquinário ou recursos financeiros - é o principal fator de produção (TZORTZAKI; MIHIOTIS, 2014).

O conhecimento passou a ter mais acentuado seu aspecto coletivo, e não mais como algo que surge inexplicavelmente em um indivíduo ou empresa isolados. Conhecimento é um fenômeno difícil de ser capturado e mensurado, ligado à capacidade de ação de quem o detém, resultado de um processo de transformação da informação em ação (BROOKING, 1996; ZELENY, 2005; SVEIBY, 2001). Nonaka e Takeuchi (1997) estenderam essa conceituação para sua aplicação no âmbito das organizações, definindo o conhecimento como resultado de um processo dinâmico, resultante da interação entre o conhecimento tácito e explícito por meio de processos sociais. Admitindo-se que o conhecimento resulta de um processo, que inclui não apenas sua criação, mas também outros fatores como a sua transferência, surge a questão de como este processo é gerido. 
Neste trabalho, não se considera o conhecimento como somente uma possessão de informação, nem somente como associado à prática. Considera-se que esses aspectos coexistem e são complementares, sendo o conhecimento uma aplicação de informações a situações específicas das empresas.

\subsection{Transferência do Conhecimento em Clusters}

O conhecimento está presente nas organizações pela soma dos conhecimentos dos indivíduos que a compõe. No nível individual, a transferência do conhecimento pode ser definida como o modo como um conhecimento adquirido em uma situação se aplica (ou falha em ser aplicada) em outra situação (SINGLEY; ANDERSON, 1989). Importante destacar que expressões como partilha de conhecimento (knowledge sharing), disseminação do conhecimento (knowledge dissemination) e transferência do conhecimento (knowledge transfer) para alguns autores são usadas como sinônimos ou aspectos intercambiáveis, enquanto outros fazem distinção entre os termos (PAULIN; SUNESON, 2015). Nesse estudo, é adotado o termo transferência do conhecimento como o processo de disseminação, compartilhamento e transferência de conhecimentos tácitos e explícitos por meio de práticas formais e informais (DAVENPORT; PRUSAK, 1998), com ênfase para a influência dos aspectos de interferência envolvidos neste processo (BITTI; ZANNI, 1993).

Organizações de uma região partilham recursos, mas também dividem conhecimento, expandindo a base de conhecimento do cluster. A vantagem do cluster pode estar baseada no quanto o conhecimento é partilhado e nos mecanismos que permitem sua disseminação, que pode ocorrer inclusive de maneira informal e sem compensação explícita. Aspectos encontrados em clusters, como velocidade na comunicação formal e informal, aliados a uma base comum de conhecimento entre as empresas, empregados e comunidade, contribuem para a criação de um ambiente favorável ao conhecimento (COOKE, 1999). Na medida em que é combinado conhecimento interno e específico com relações entre os membros do cluster, aumenta-se a capacidade de absorção do conhecimento, que flui além das fronteiras do cluster (TALLMAN et al, 2004). Desta forma, uma rede de conhecimento se constitui no cluster por meio de uma estrutura de links entre os atores, que facilita o aprendizado e a transferência (TALLMAN et al, 2004). As diferentes formas de redes de conhecimento incluem subcontratações, cadeias de suprimentos, colaborações formais e informais, relações entre empregados e familiares, participação em associações comercias, parcerias e movimentação de pessoas entre as empresas em diferentes níveis (ZENG et al, 2008).

Portanto, uma rede de conhecimento é uma estrutura de atores interconectados que 
facilitam o aprendizado nas organizações. Para o presente trabalho a transferência do conhecimento é o processo de disseminação, compartilhamento e transferência de conhecimentos tácitos e explícitos por meio de práticas formais e informais (DAVENPORT; PRUSAK, 1998; BITTI; ZANNI, 1993), obtido por meio de uma rede existente, assim entendida como uma estrutura de links entre os atores que permitem o aprendizado (TALLMAN et al, 2004). O cluster não apenas fornece suporte ao desenvolvimento econômico, mas atua como facilitador ou inibidor do processo de criação e transferência do conhecimento. Embora o conhecimento seja transferido de forma mais rápida entre organizações atuando em rede do que empresas isoladas, sua análise envolve maior complexidade (DARR; ARGOTE; EPPLE, 1995; POWELL; KOPUT; SMITH-DOERR, 1996), em um tema de difícil mensuração.

\subsection{Fatores do processo de transferência do conhecimento}

Mais do que discutir os componentes e terminologias envolvidas no processo de transferência do conhecimento, é importante considerar os fatores que influenciam esse processo. Szulanski $(1996,2000)$ descreveu uma série de inibidores da transferência do conhecimento ao explicar porque conhecimentos e habilidades podem ser difíceis de serem transmitidos entre pessoas e organizações. Paulin e Winroth (2013) discutiram a transferência do conhecimento como um processo influenciado por fatores facilitadores e fatores inibidores, se baseando na ideia de que o processo pode ser influenciado de diferentes formas, envolvendo um ator como sendo a fonte do conhecimento, que tem a motivação de transmitir a um ator receptor. Este processo é também influenciado pelo contexto em que ocorre.

$\mathrm{Na}$ dimensão da transferência do conhecimento em cluster verifica-se que embora o conhecimento esteja disponível, nem todos os membros o percebem e tem o mesmo acesso e quando o fazem, não é de forma simétrica. Há diferença no processo de transferência do conhecimento entre os diferentes níveis existentes em um cluster, tais como na relação fornecedores e produtores, empresas líderes e pequenas empresas (SARACH, 2015). As relações existentes no cluster, sejam de cooperação ou não, intensificam a troca de conhecimento entre os participantes. Mesmo quando não é reconhecida a existência de cooperação entre os membros do cluster a transferência de conhecimento ocorre (HOFFMANN; LOPES; MEDEIROS, 2014).

O processo de transferência de conhecimento no cluster tem dimensões distintas, tais como cooperação, relação com instituições de apoio e mobilidade da força de trabalho, sendo multidimensional ao envolver troca de técnicas, partilha de informações e complementaridade entre os membros (BENTON, 1993; ASHEIM; ISAKSEN, 2002; ZENG et al, 2008). Dentre as relações de cooperação que surgem no cluster, autores destacaram a importância das relações sociais 
informais, inclusive familiares, no processo de transferência do conhecimento em regiões em que existe o cluster (MOLINA-MORALES, 2001; BASANT, 2002; MOROSINI, 2004). De forma paradoxal, no cluster existe a coexistência de colaboração e competição, que no aspecto da transferência do conhecimento se manifesta no receio de perda de competitividade e risco de oportunismo ao se partilhar o conhecimento com outras empresas (BRUSCO, 1990; JARVENPAA; WERNICK, 2011). Relativo à dimensão da mobilidade da força de trabalho, um cluster tem a capacidade de reunir em um mesmo local um número significativo de pessoas com habilidades específicas. Portanto, a movimentação da mão de obra, junto com a proximidade geográfica, influenciam a transferência do conhecimento (BASANT, 2002; ASHEIM; ISAKSEN, 2002; MALMBERG; POWER, 2005).

\section{METODOLOGIA}

Este trabalho propõe um estudo exploratório, em relação aos seus objetivos (GIL, 2008), pois permite uma visão geral sobre determinado tema de pesquisa pouco explorado, o que torna difícil a formulação de hipóteses precisas e operacionalizáveis. A lógica do estudo é indutiva (RICHARDSON, 1999), pois parte de dados ou observações particulares constatadas visando a obter proposições gerais, não contidas nas partes examinadas. Neste estudo, é proposto um modelo conceitual para estudos sobre transferência do conhecimento em cluster, a partir de um estudo bibliográfico (GIL, 2008), a partir da coleta e análise de dados secundários, decorrentes de publicações científicas (Figura 1).

A investigação conduzida, orientada para o levantamento das bases para proposição de modelo conceitual para estudo de transferência do conhecimento em cluster, usando aspectos da perspectiva de redes, foi desenvolvida a partir de painel de artigos do período de 2011 a 2016 sobre transferência do conhecimento, redes e cluster que, juntamente com os fundamentos teóricos apresentados no item anterior, compõem o modelo proposto. Para a investigação da produção internacional foi utilizado o Portal Proquest, devido a seu reconhecimento como depositório de produção acadêmica internacional. Para a pesquisa da produção acadêmica brasileira foi utilizado o portal Scielo, devido ao número de trabalhos que podem ser encontrados neste portal por meio de acesso livre, complementada com pesquisa no portal Spell, que permitia a restrição da busca a periódicos considerados mais relevantes ao problema proposto neste trabalho, além de disponibilizar trabalhos do período de 2016. Os resultados de análises bibliográficas indicam a predominância de estudos voltados ao desempenho do cluster, principalmente no aspecto da inovação, bem como estudos de fatores isolados envolvendo o processo de transferência do conhecimento. 
Figura 1 - Delimitação da pesquisa

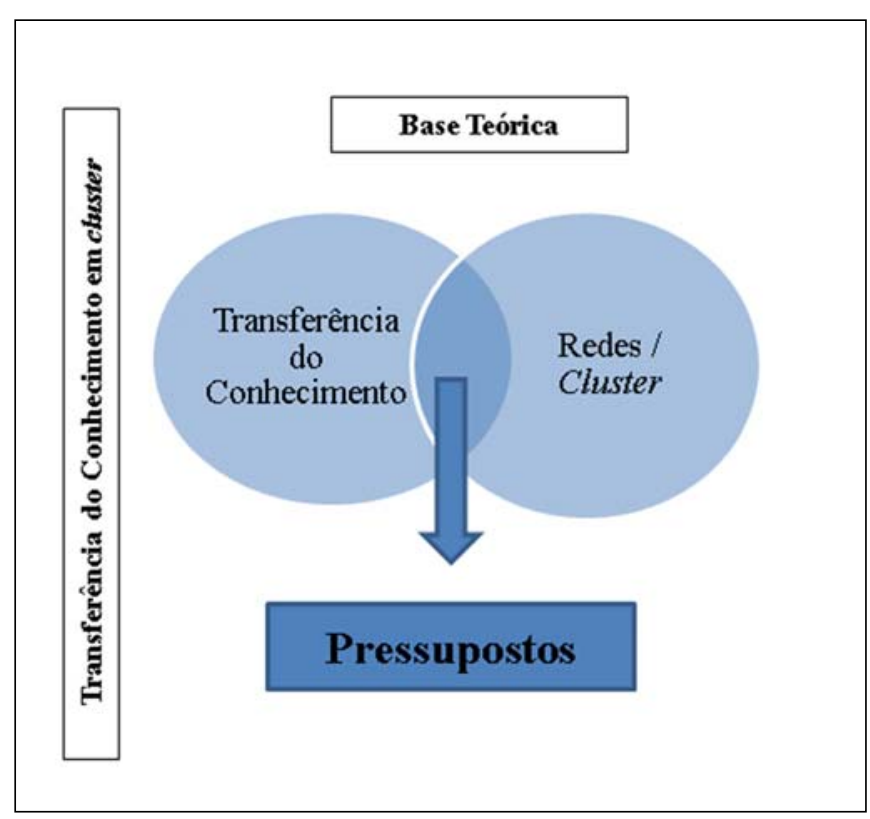

Fonte: elaborado pelos autores (2016).

$\mathrm{Na}$ investigação da produção acadêmica foram buscados artigos em periódicos acadêmicos, por serem trabalhos cujo processo de avaliação para publicação é mais criterioso, sobre os temas delimitados na Figura 1. No portal Proquest foram encontrados 868.980 artigos em periódicos acadêmicos, sendo aproximadamente 73.000 estudos de redes em administração, equivalente a aproximadamente $8 \%$ da produção. Tal incidência também pode ser encontrada no tema conhecimento, cujos trabalhos encontrados na área de administração representam aproximadamente $16 \%$ da produção. Indica-se, assim, haver campo para investigação sobre os conceitos de redes e conhecimento aplicados aos estudos organizacionais. Prosseguindo a pesquisa da produção internacional, foram adicionadas palavras-chaves de expressões relativas ao tema redes: 1) Network; 2) Networks; 3) Inter-organizational networks; 4) Business network, optandose por escolher a que apresentasse maior número de indicações, que foram network e business network.

Dentre os trabalhos encontrados, foi delimitado o período de tempo para a última década e, em seguida, para os últimos 05 anos, com o intuito de manter a coerência e atualidade entre o tema e os objetivos do trabalho, ampliando-se a pesquisa incluindo as palavras-chaves: network, business network, cluster, knowledge, organizational knowledge, knowledge managment, knowledge transfer, no título dos trabalhos, no período de 2011 a 2016, período que apresentou dados mais significativos sobre os temas, cujos dados são resumidos no Quadro 1. 
Quadro 1- Produção acadêmica internacional - Redes, Cluster e Conhecimento - Proquest

\begin{tabular}{|l|c|c|c|}
\hline \multicolumn{1}{|c|}{ Palavra } & Total & $\begin{array}{c}\text { Período de } \\
\mathbf{2 0 0 6} \text { a 2016 }\end{array}$ & $\begin{array}{c}\text { Período de } \\
\mathbf{2 0 1 1} \text { a 2016 }\end{array}$ \\
\hline Network (1) & 868.980 & 542.100 & 337.525 \\
\hline Business network (2) & 98.929 & 57.420 & 38.761 \\
\hline Cluster (3) & 204.504 & 118.209 & 153.215 \\
\hline Knowledge (4) & 488.188 & 276.052 & 4.987 \\
\hline Organizational Knowlege (5) & 16.143 & 10.045 & 30.028 \\
\hline Knowledge Management (6) & 90.885 & 56.587 & 5.631 \\
\hline Knowledge Transfer (7) & 16.516 & 10.252 & 15.917 \\
\hline Network, Knowledge (1) e (4) & 39.169 & 26.456 & 650 \\
\hline Network, Knowledge Transfer (1), (7) & 1.591 & 1.130 & 1.010 \\
\hline Business Network, Cluster (2) e (3) & 1.662 & 1.346 & \\
\hline Cluster, Knowledge Transfer (3) e (7) & 233 & 175 & \\
\hline
\end{tabular}

Fonte: elaborado pelos autores (2016).

Para pesquisa da produção acadêmica brasileira foi utilizado o portal Scielo, complementada com pesquisa no portal Spell. Verifica-se que no período de 2005 a 2015 são encontradas 1.961 publicações sobre o tema de redes, sendo 1.074 publicações dos últimos cinco anos, indicando que o tema passou a ser mais pesquisado. Utilizando a palavra conhecimento são encontradas 8.718 publicações nacionais na última década, sendo que 4.530 publicações se referem ao período de 2011 a 2015 sugerindo, assim, que o assunto também passou a ser mais intensamente investigado, ambos em uma proporção equivalente ao encontrado nas indicações internacionais. De modo a manter a atualidade e coerência da pesquisa foi considerado apenas o período de 2011 a 2015 e restringiu-se a trabalhos que contivessem as palavras-chaves no título, nos idiomas português e inglês, sendo utilizadas as palavras-chave redes: redes interorganizacionais, cluster, conhecimento, conhecimento organizacional e transferência do conhecimento, cujos resultados estão no Quadro 2. 
Quadro 2 - Produção acadêmica brasileira - Redes, Cluster e Conhecimento

\begin{tabular}{|c|c|c|c|c|}
\hline \multirow{2}{*}{ Palavra } & \multicolumn{2}{|c|}{ Frequência - Scielo } & \multicolumn{2}{|c|}{ Frequência - Spell } \\
\hline & Total & $\begin{array}{c}2011 \text { a } \\
2015\end{array}$ & Total & $\begin{array}{c}2011 \text { a } \\
2016\end{array}$ \\
\hline Redes (1) & 2.315 & 1.074 & 1.154 & 599 \\
\hline Redes Interorganizacionais (2) & 44 & 20 & 129 & 68 \\
\hline Cluster (3) & 1.785 & 783 & 422 & 208 \\
\hline Conhecimento (4) & 11.128 & 4.530 & 2.544 & 1.332 \\
\hline Conhecimento Organizacional (5) & 202 & 89 & 496 & 253 \\
\hline Gestão do Conhecimento (6) & 579 & 277 & 1.198 & 676 \\
\hline Transferência do Conhecimento (7) & 131 & 66 & 102 & 55 \\
\hline Redes e Conhecimento (1) e (4) & 192 & 103 & 49 & 32 \\
\hline Redes, Conhecimento, Transferência & 05 & 03 & 06 & 04 \\
\hline Redes e Cluster (1) e (3) & 30 & 12 & 22 & 12 \\
\hline Cluster e Conhecimento (3) e (4) & 60 & 28 & 12 & 06 \\
\hline Conhecimento e Transferência & 138 & 68 & 40 & 22 \\
\hline
\end{tabular}

Fonte: elaborado pelos autores (2016).

Pelos dados do Quadros 2 se verifica que, embora tenha havido aumento no interesse sobre os temas tratados neste trabalho, a relação entre redes e conhecimento foi pouco investigada na produção acadêmica brasileira nos últimos anos, assim como o processo de transferência do conhecimento como foco de pesquisa. Essa intensificação do interesse é acentuada nas diferentes combinações dos temas redes, conhecimento e cluster nos últimos anos, indicando que essa intersecção de fatores tem sido pesquisada mais recentemente. Assim, são corroborados os indícios encontrados na análise do Quadro 1, ou seja, de que, embora tenha havido aumento no interesse sobre os temas tratados neste trabalho, a relação entre redes e conhecimento foi pouco investigada na produção acadêmica brasileira nos últimos anos, assim como o processo de transferência do conhecimento como foco de pesquisa. Também nota-se uma escassez de trabalhos que investiguem o processo de transferência de conhecimento em cluster, embora este aspecto tenha sido relatado frequentemente na literatura. Em relação à pesquisa na produção nacional foi preciso ampliar a pesquisa incluindo também trabalhos que contivessem essas palavras-chave em seu resumo e não apenas nos títulos, devido à escassez de trabalhos obtidos, também voltados em sua maioria para o tema da inovação ou para a transferência do conhecimento intraorganizacional, ou seja, dentro dos limites da organização como unidade de análise isolada. Os trabalhos existentes, em sua maioria, tratam ainda do aglomerado de empresas e o reconhecimento dessa forma de atuação por parte do consumidor. É possível também identificar que, na temática de gestão do conhecimento, o enfoque dos autores tem sido intraorganizacional, ou seja, a preocupação é no processo do conhecimento dentro dos limites da organização. 
Em continuidade à revisão bibliográfica, foi efetuada a análise dos títulos e resumos dos trabalhos obtidos na pesquisa resumida nos Quadros 1 e 2, do período de 2011 a 2016, bem como excluídos os estudos que abordam aspectos não pertinentes ao presente estudo, tais como trabalhos das áreas de informática, saúde, biotecnologia, educação, estratégia, gestão de sistemas bancários e marketing. Depois de aplicados os critérios de restrição, as abordagens e principais contribuições serão relatadas a seguir.

\section{ANÁLISE DOS RESULTADOS}

Connell e Voola (2013) buscaram investigar como os membros de um cluster industrial partilhavam conhecimento na perspectiva de redes, de modo a aumentar sua vantagem competitiva, por meio de um estudo utilizando conceitos de marketing. Os autores verificaram que a partilha de conhecimento e colaboração entre os membros do cluster requer facilitação ativa, principalmente quando são introduzidos novos membros. Castro (2015) buscou enfatizar a relação de cooperação entre atores localizados na mesma área geográfica, com interdependência econômica e cultura distinta, demonstrando como o conhecimento ultrapassa fronteiras por meio de estruturas dinâmicas e colaborativas. $\mathrm{O}$ autor defende que essas atividades têm seus resultados ampliados quando emergem do sistema de governança, cujo processo de maturidade coincide com a maturidade do cluster. Sarach (2015) analisou as relações de cooperação em cluster industrial, no contexto da inovação e transferência do conhecimento, enfatizando que o desenvolvimento sustentável de um cluster pode aumentar a competitividade global de uma região.

Os resultados encontrados mostram a necessidade de aumentar a cooperação entre os membros do cluster para potencializar a transferência do conhecimento, principalmente na relação entre a empresa líder e universidades. De modo diverso, Hoffmann, Lopes e Medeiros (2014) encontraram evidências de que a transferência de conhecimento ocorre em um cluster mesmo quando não há relações de cooperação reconhecida pelos seus membros. O estudo trouxe ainda evidências de que apenas a presença física em um cluster não assegura o acesso ao processo de transferência de conhecimento. No contexto da gestão do conhecimento em Parques Tecnológicos, Vásquez-Urriago, Barge-Gil e Rico (2016) analisaram o processo de cooperação para a inovação, com ênfase nos efeitos da localização em um parque de ciências e tecnologia, na cooperação para inovação e nos mecanismos que afetam esse processo. As evidências encontradas sugerem que resultados de cooperação e inovação são maiores em empresas localizadas em Parques Tecnológicos, uma vez que a proximidade propicia o desenvolvimento de relações mais 
colaborativas, e não deve ser entendida apenas geograficamente, mas tecnológica e organizacional (estrutura, cultura e linguagem).

Parte dos trabalhos que buscam relacionar gestão do conhecimento e redes se concentram na relação entre empresas e universidades, com foco para a busca do conhecimento com o objetivo final da inovação (ARVANITIS; KUBLI; WOERTER, 2011; GRIMPE; HUSSINGER, 2013). Outros trabalhos buscaram relacionar se as empresas localizadas em um cluster tem mais capacidade de absorver o conhecimento externo, uma vez que atuam em um ambiente com alto nível de transferência do conhecimento. Cheng, Niu e Niu (2014) examinaram as relações entre empresas de um cluster industrial, seu aprendizado organizacional e sua habilidade em se adaptar ao ambiente externo, cujos resultados indicam que estar situado em um cluster industrial aumenta o aprendizado das empresas. Os autores sustentam que organizações participantes de redes, como um cluster industrial, no qual há um alto nível de conhecimento e habilidades de transformação, são mais propensos a absorver com sucesso o conhecimento externo. Já Berbegal-Mirabent, Lafuente e Sole (2013) estudaram o impacto do exercício de atividades de transferência de conhecimentos em universidades, buscando incorporar conceitos de cluster à análise. Os autores realizaram uma investigação do desempenho das universidades, sendo considerados como indicadores de desempenho número de patentes e spin-off (comercialização de pesquisas para o mercado), avaliando a relação entre eficiência e intensidade da transferência do conhecimento.

O estudo de Berbegal-Mirabent, Lafuente e Sole (2013) utilizou uma perspectiva multidimensional por utilizar uma análise de cluster para complementar a avaliação. Entretanto, o termo cluster foi utilizado com o sentido de região ou território, já que os autores buscaram considerar a diferença de resultado entre as regiões estudadas. Os resultados revelaram que fatores regionais relacionados ao desenvolvimento tecnológico e cultura empresarial influenciam significativamente a eficiência das universidades e seu envolvimento em atividades de transferência do conhecimento. Dentre os estudos nacionais, Sohn et al (2014) buscaram identificar e analisar os canais de aprendizagem em cluster têxtil e de vestuário, realizando um estudo comparativo entre um cluster localizado em Santa Catarina, no Brasil, e outro na região norte de Portugal. A pesquisa dos autores revelou que no cluster brasileiro são rarefeitos os relacionamentos entre as empresas e com as universidades, assim como a colaboração não é reconhecida como importante para a transferência do conhecimento que levaria as empresas à inovação.

Verifica-se uma predominância de estudos com foco para a inovação, ao tratarem da intersecção entre gestão do conhecimento e cluster. Há também a predominância de estudos de fatores isolados do processo de transferência do conhecimento, tais como cooperação ou relações 
sociais e o impacto para o desempenho do cluster. Nesse sentido, pretende-se apresentar uma proposta de modelo conceitual para estudo do processo de transferência do conhecimento entre organizações participantes de cluster. O estudo dos fundamentos da gestão do conhecimento (tipos de conhecimento, abordagens de gestão do conhecimento, transferência do conhecimento), fatores que interferem no processo da transferência do conhecimento (facilitadores e inibidores) e dos aspectos existentes em cluster que o diferenciam de demais formatos organizacionais forneceram suporte à definição dos pressupostos teóricos para estudos sobre transferência do conhecimento em cluster.

Apresenta-se assim o modelo conceitual para estudo do processo de transferência do conhecimento entre organizações participantes de cluster. Neste trabalho, o termo pressuposto é entendido como uma forma de expressar parâmetros básicos que servem de caminho e de baliza na interação com a realidade empírica (MINAYO, 2000). Foram delineados quatro pressupostos e fatores que podem facilitar ou inibir a transferência do conhecimento no cluster.

O processo de transferência de conhecimento no cluster tem dimensões distintas (cooperação, relação com instituições de apoio, mobilidade da força de trabalho e proximidade espacial), conforme indicado por Benton (1993), Molina-Morales (2001), Asheim e Isaksen (2002), Basant (2002), Morosini (2004), Malmberg e Power (2005), Zeng et al (2008). A cooperação, no contexto da transferência do conhecimento, envolve a troca de técnicas, partilha de informações e complementaridade entre os membros do cluster (BENTON, 1993; ASHEIM; ISAKSEN, 2002; ZENG et al, 2008). Dentre as relações de cooperação que surgem no cluster, autores destacaram a importância das relações sociais informais, inclusive familiares, no processo de transferência do conhecimento em regiões em que existe o cluster (MOLINA- MORALES, 2001; BASANT, 2002; MOROSINI, 2004).

Outro aspecto a ser considerado é que a atuação em um cluster pode fazer surgir o receio de comportamento oportunista e o risco de perda de competitividade, influenciando a partilha de informações e conhecimento (JARVENPAA; WERNICK, 2011). Assim, de forma paradoxal, no cluster existe a coexistência de colaboração e competição (HOFFMANN; LOPES; MEDEIROS, 2014), que no aspecto da transferência do conhecimento se manifesta no receio de perda de competitividade e risco de oportunismo ao se partilhar o conhecimento com outras empresas (BRUSCO, 1990; JARVENPAA; WERNICK, 2011). O primeiro pressuposto (Quadro 3) diz respeito às relações de cooperação que podem ou não ocorrer entre as organizações de um cluster e foi assim enunciado:

\section{A transferência do conhecimento no cluster é influenciada pelos aspectos de cooperação.}


Quadro 03 - Pressuposto 1 (Cooperação)

\begin{tabular}{|c|c|c|}
\hline Fatores & Característica & Autores \\
\hline Credibilidade & Facilitador/Inibidor & $\begin{array}{l}\text { Szulanski (1996); Arvanitis, } \\
\text { Kubli e Woerter (2011) }\end{array}$ \\
\hline $\begin{array}{c}\text { Troca de técnicas e novas } \\
\text { tecnologias }\end{array}$ & Facilitador /Inibidor & \multirow{4}{*}{$\begin{array}{l}\text { Benton (1993); Asheim e } \\
\text { Isaksen (2002); Zeng et al } \\
\text { (2008); Arvanitis, Kubli e } \\
\text { Woerter (2011); Hoffmann, } \\
\text { Lopes e Medeiros (2014) }\end{array}$} \\
\hline Relações estabelecidas & Facilitador/Inibidor & \\
\hline Partilha de informações & Facilitador /Inibidor & \\
\hline $\begin{array}{c}\text { Complementaridade de serviços e } \\
\text { recursos }\end{array}$ & Facilitador /Inibidor & \\
\hline Receio da perda de competitividade & Inibidor & $\begin{array}{l}\text { Brusco (1990); Jarvenpaa e } \\
\text { Wernick (2011) }\end{array}$ \\
\hline $\begin{array}{l}\text { Receio de estar exposto a } \\
\text { oportunismo }\end{array}$ & Inibidor & $\begin{array}{l}\text { Brusco (1990); Jarvenpaa e } \\
\text { Wernick (2011) }\end{array}$ \\
\hline
\end{tabular}

Fonte: elaborada pelos autores (2016).

Já o segundo pressuposto (Quadro 4) envolve as relações entre empresas e instituições de apoio que podem compor estruturas organizacionais tais como parques tecnológicos, incubadoras de empresa, polos tecnológicos e industriais. Em um cluster pode existir a atuação de instituições de apoio, que proveem suporte e assistência aos participantes. Essas instituições podem ser de natureza pública, tais como universidades, ou associações privadas responsáveis pela governança do cluster. As relações estabelecidas com essas instituições de apoio podem ocorrer por meio do conhecimento técnico, acesso a especialistas, fornecimento de mão de obra com qualificação específica, oferecimento de cursos e realização de eventos de disseminação do conhecimento (ASHEIM; ISAKSEN, 2002; HOFFMANN; LOPES; MEDEIROS, 2014).

Foi assim enunciado o segundo proposto: A transferência do conhecimento no cluster é influenciada pela relação com instituições (instituições de apoio, instituições de ensino e pesquisa).

Quadro 04 - Pressuposto 2 (Relação com Instituições)

\begin{tabular}{|c|c|c|}
\hline Fatores & Característica & Autores \\
\hline Acesso ao conhecimento técnico & Facilitador & \multirow{5}{*}{$\begin{array}{l}\text { Asheim e Isaksen (2002); } \\
\text { Etzkowitz (2003); Arvanitis, } \\
\text { Kubli e Woerter (2011); } \\
\text { Hoffmann, Lopes e Medeiros } \\
\text { (2014) }\end{array}$} \\
\hline Acesso a especialistas & Facilitador & \\
\hline $\begin{array}{c}\text { Treinamentos e cursos específicos } \\
\text { de interesse do cluster }\end{array}$ & Facilitador / Inibidor & \\
\hline Mão de obra qualificada & Facilitador & \\
\hline Eventos/Congressos/Seminários & Facilitador / Inibidor & \\
\hline $\begin{array}{c}\text { Projetos realizados em parceria } \\
\text { (pesquisas, artigos, teses e } \\
\text { dissertações) }\end{array}$ & Facilitador / Inibidor & \multirow[t]{2}{*}{$\begin{array}{l}\text { Etzkowitz (2003); Arvanitis, } \\
\text { Kubli e Woerter (2011); Rubin, } \\
\text { Aas e Stead (2015). }\end{array}$} \\
\hline Diferença de objetivos e conflito & Inibidor & \\
\hline
\end{tabular}




\begin{tabular}{|c|l|l|}
\hline $\begin{array}{c}\text { de interesses } \\
\text { Discussões sobre propriedade } \\
\text { intelectual }\end{array}$ & \multicolumn{2}{|}{} \\
\cline { 1 - 2 } Propensão menor a riscos & Inibidor & \\
\hline $\begin{array}{c}\text { Diferença do nível de } \\
\text { conhecimento }\end{array}$ & Inibidor & $\begin{array}{l}\text { Arvanitis, Kubli e Woerter } \\
(2011) ; \text { Noveli e Segatto } \\
(2012) .\end{array}$ \\
\hline
\end{tabular}

Fonte: elaborado pelos autores (2016).

O terceiro pressuposto (Quadro 5) diz respeito ao impacto da mobilidade da força do trabalho, aspecto encontrado de forma proeminente entre organizações que compartilham o espaço geográfico típico de um cluster. Com relação à dimensão da mobilidade da força de trabalho, esse aspecto já havia sido destacado por Marshall (1890), ao comentar que um cluster tem a capacidade de reunir em um mesmo local um número significativo de pessoas com habilidades específicas. Assim, a movimentação da mão de obra entre as empresas do cluster, entre a universidade e as empresas, em conjunto com a proximidade geográfica, influenciam a transferência do conhecimento (BASANT, 2002; ASHEIM; ISAKSEN, 2002; MALMBERG; POWER, 2005). O terceiro pressuposto é assim enunciado: A transferência do conhecimento no cluster é influenciada pela mobilidade da força de trabalho.

Quadro 05 - Pressuposto 3 (Mobilidade da Força de Trabalho)

\begin{tabular}{|c|c|c|}
\hline Fatores & Característica & Autores \\
\hline $\begin{array}{l}\text { Empregados com experiência } \\
\text { anterior da mão de obra em outras } \\
\text { organizações do cluster }\end{array}$ & Facilitador & \multirow{4}{*}{$\begin{array}{l}\text { Basant (2002); Asheim e } \\
\text { Isaksen (2002); Malmberg e } \\
\text { Power (2005); Arvanitis, Kubli } \\
\text { e Woerter (2011); Hoffmann, } \\
\text { Lopes e Medeiros (2014). }\end{array}$} \\
\hline $\begin{array}{c}\text { Consultores com experiência } \\
\text { anterior em outras organizações } \\
\text { do cluster }\end{array}$ & Facilitador & \\
\hline $\begin{array}{c}\text { Fornecedores com experiência } \\
\text { anterior em outras organizações } \\
\text { do cluster }\end{array}$ & Facilitador & \\
\hline $\begin{array}{l}\text { Formação anterior nas instituições } \\
\text { de ensino do cluster }\end{array}$ & Facilitador & \\
\hline
\end{tabular}

Fonte: elaborado pelos autores (2016).

O quarto pressuposto (Quadro 6) diz respeito ao impacto da proximidade típica de um cluster no processo de transferência do conhecimento, em relação aos seus aspectos estruturais e culturais. A proximidade geográfica existente no cluster intensifica a troca de conhecimento entre uma organização para outra, fenômeno este já observado por Marshall (1890). Uma rede de conhecimento se constitui no cluster por meio de uma estrutura de links entre os atores, que facilita o aprendizado e a transferência do conhecimento nessa rede (TALLMAN et al, 2004), levando ao transbordamento do conhecimento, independente de pertencer ou não à mesma unidade 
organizacional a que o agente pertence (CORREIA, 2007; VÁSQUEZ-URRIAGO; BARGE-GIL; RICO, 2016). Aspectos encontrados em clusters como velocidade na comunicação formal e informal, aliada a uma base comum de conhecimento entre as empresas, empregados e comunidade, contribuem para a criação de um ambiente favorável ao conhecimento (COOKE, 1999; CASTRO, 2015). Assim, um processo de aprendizado coletivo se dá no cluster por meio de uma rede intrincada de contatos informais em encontros presenciais, fluxo de informações casuais (CHENG; NIU; NIU, 2014), gerando um comportamento social orientado para o compartilhamento de valores e normas de conduta, envolvendo aspectos profissionais e pessoais (ZACCARELLI et al, 2008). Apresenta-se assim o quarto e último pressuposto, sendo assim enunciado: $\mathbf{A}$ transferência do conhecimento no cluster é influenciada pela proximidade.

Quadro 06 - Pressuposto 4 (Proximidade)

\begin{tabular}{|c|c|c|}
\hline Fatores & Característica & Autores \\
\hline Proximidade geográfica & Facilitador / Inibidor & \multirow{2}{*}{$\begin{array}{l}\text { Etzkowitz (2003); Zaccarelli et } \\
\text { al (2008); Paulin e Winroth } \\
\text { (2013). }\end{array}$} \\
\hline $\begin{array}{l}\text { Existência de espaços físicos } \\
\text { adequados ao aprendizado }\end{array}$ & Facilitador/Inibidor & \\
\hline $\begin{array}{l}\text { Compreensão do cenário de } \\
\text { aplicação do conhecimento }\end{array}$ & Facilitador/Inibidor & $\begin{array}{l}\text { Szulanski (1996); Arvanitis, } \\
\text { Kubli e Woerter (2011). }\end{array}$ \\
\hline Disponibilidade de tempo & Facilitador/Inibidor & $\begin{array}{l}\text { Szulanski (1996); Arvanitis, } \\
\text { Kubli e Woerter (2011); Paulin } \\
\text { e Winroth (2013) }\end{array}$ \\
\hline $\begin{array}{c}\text { Canais de Comunicação: informais } \\
\text { (eventos, festas, encontros } \\
\text { familiares) e formais (contratos, } \\
\text { licenças) }\end{array}$ & Facilitador /Inibidor & $\begin{array}{lcr}\text { Molina- } & \text { Morales } & (2001) ; \\
\text { Basant } \quad(2002) ; & \text { Morosini } \\
\text { (2004); Hoffmann, Lopes e } \\
\text { Medeiros (2014). }\end{array}$ \\
\hline $\begin{array}{c}\text { Proximidade cultural (linguagem } \\
\text { em comum, crenças e valores } \\
\text { partilhados) }\end{array}$ & Facilitador /Inibidor & $\begin{array}{l}\text { Siegel, Waldman e Link } \\
\text { (2003); Zaccarelli et al (2008); } \\
\text { Abramovsky e Simpson } \\
\text { (2011). }\end{array}$ \\
\hline
\end{tabular}

Fonte: elaborado pelos autores (2016)

Verifica-se que um mesmo fator pode atuar facilitando ou restringindo a transferência do conhecimento. A credibilidade entre as organizações, por exemplo, pode aumentar a disposição entre os envolvidos para atuar em projetos em parceria. Já a ausência de credibilidade, por outro lado, pode inibir a transferência do conhecimento na medida em que diminui a disposição entre organizações de atuarem em conjunto. A proteção ao oportunismo pode intensificar a cooperação entre organizações, da mesma forma que o receio de estar sujeito ao oportunismo, ao compartilhar informações, pode inibir o processo de transferência do conhecimento entre as organizações inseridas no cluster. Essa premissa pode ser sintetizada pela Figura 2, suportado pelos conceitos envolvidos na transferência do conhecimento em cluster, explicitados na fundamentação teórica, cuja terminologia se baseia na ideia de que o processo de transferência do conhecimento pode ser 
influenciado de diferentes formas, podendo ser por meio de fatores facilitadores (que influenciam positivamente), fatores inibidores (que influenciam negativamente) ou obstáculos (que obstruem o processo).

Figura 2-Modelo Conceitual para pesquisa de transferência do conhecimento em cluster

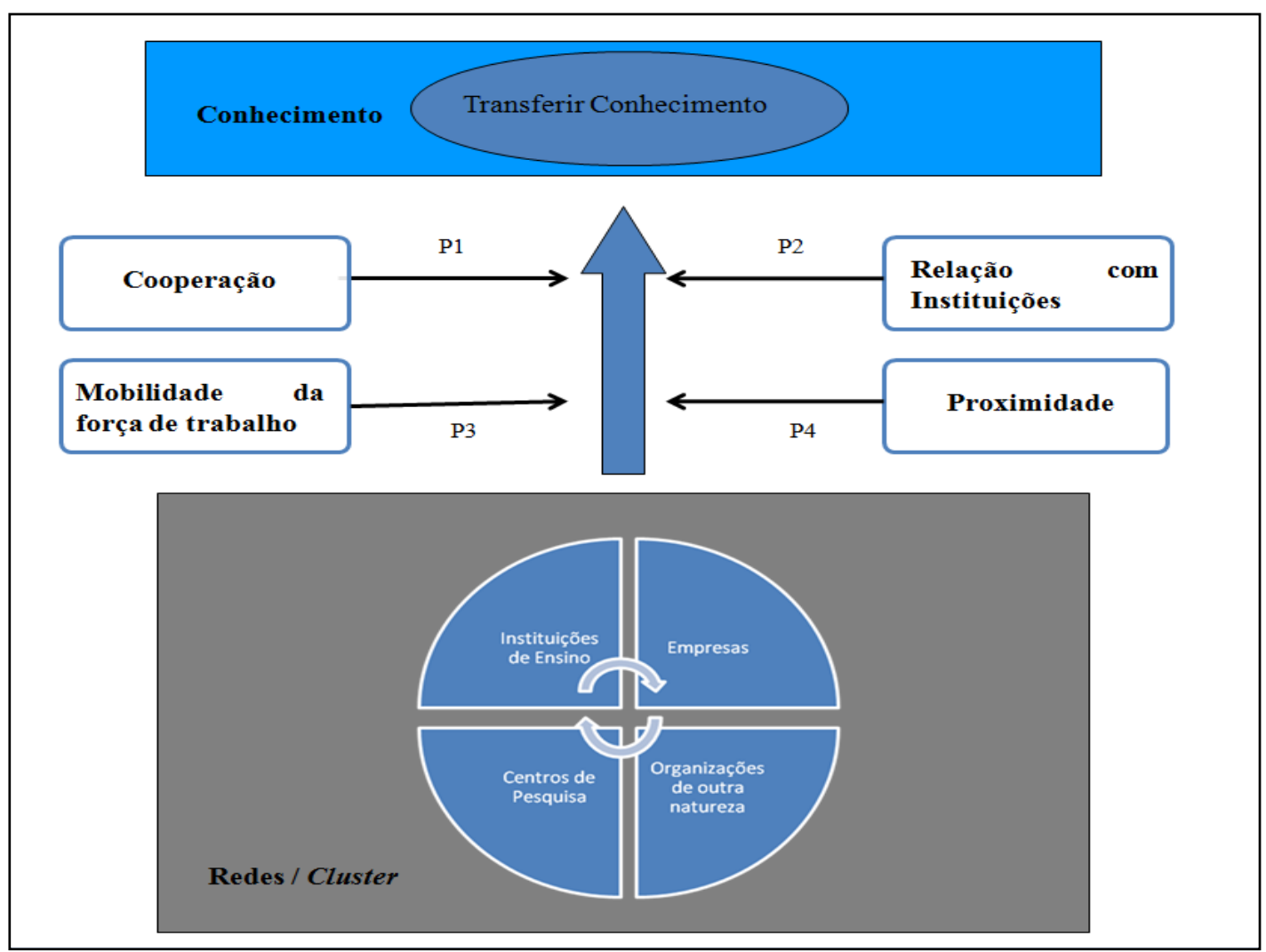

Fonte: elaborado pelos autores (2016)

Estes pressupostos representados pela Figura 2 delimitam o esquema conceitual que pode atuar como direcionador em estudos sobre transferência do conhecimento entre as organizações inseridas no cluster, bem como fornecem suporte aos gestores das empresas e organizações públicas que tenham o objetivo de explorar o potencial dos clusters para o desenvolvimento econômico regional.

\section{CONCLUSÃO}

É possível afirmar que a transferência do conhecimento sofre a influência de diferentes fatores, que podem impactar este processo, na medida em que este processo entre as organizações inseridas em cluster tem dimensões distintas (cooperação, relação com instituições de apoio, mobilidade da força de trabalho e proximidade espacial), ou seja, o processo de transferência do conhecimento em um cluster tem um aspecto multidimensional. Desta forma, apesar do potencial para a criação e disseminação do conhecimento que reconhecidamente existe 
entre organizações inseridas em um cluster, existem fatores que podem atuar como facilitadores, influenciando positivamente a transferência do conhecimento, bem como existem fatores que se constituem uma restrição, influenciando negativamente o processo. Este trabalho tem por objetivo apresentar uma proposta de modelo conceitual para estudo do processo de transferência do conhecimento entre as organizações participantes de cluster, por meio do qual podem ser identificados os fatores que influenciam, tanto positivamente quanto de forma negativa, este processo.

A partir do levantamento teórico de trabalhos acadêmicos sobre transferência do conhecimento, redes e cluster, foi proposto um modelo conceitual para estudos sobre o tema, instituído a partir de quatro pressupostos, diante da ausência de consenso quanto aos fatores que podem interferir na transferência do conhecimento no cluster. Verifica-se que, contexto de redes interorganizacionais e clusters, a transferência do conhecimento conduz ao desafio que é a implantação de mecanismos que facilitem a transferência do conhecimento, bem como a erradicação e fatores que podem inibir este processo. Apesar da diferença de terminologia e classificações, os autores convergem quanto a existirem fatores de diferentes naturezas que podem influenciar o processo de transferência do conhecimento nas organizações, tanto no sentido de facilitar quanto no sentido de restringir esse processo. Conclui-se, portanto, que no contexto de redes interorganizacionais e clusters a transferência do conhecimento conduz ao desafio que é a implantação de mecanismos que facilitem a transferência do conhecimento, bem como a erradicação e fatores que podem inibir este processo.

Este trabalho pretende assim apresentar uma ferramenta metodológica que possa orientar os pesquisadores na realização de estudos sobre clusters. Apresenta ainda uma contribuição prática gerencial, na medida em que o modelo proposto pode orientar gestores em estratégias que visem explorar o potencial dos clusters. Uma das limitações existentes neste trabalho é de natureza metodológica, quanto à dificuldade de definição de conceitos, pois o tema tangencia áreas como inovação e gestão da informação. Outra limitação deste trabalho é a ausência de aplicação empírica do modelo proposto para validação. Recomenda-se que estudos futuros apliquem o modelo em contexto empírico, bem como sejam feitos estudos em clusters de diferentes naturezas e regiões, para estudos comparativos. 


\section{REFERÊNCIAS}

ABRAMOVSKY, L.; SIMPSON, H. Geographic proximity and firm-university innovation linkages: evidence from Great Britain. Journal of Economic Geography, v. 11, p. 949-977, 2011.

ALLEN, T.J. Managing the flow of technology: technology transfer and the dissemination of technological information within the R and D organization. Cambridge: Massachusetts Institute of Technology, 1977.

AMATO NETO, J. Redes de cooperação produtiva e clusters regionais oportunidades para as pequenas e médias empresas. São Paulo: Atlas; Fundação Vanzolini, 2000.

ARVANITIS, S.; KUBLI, U.; WOERTER, M. Knowledge and Technology Transfer Activities between Firms and Universities in Switzerland: An Analysis Based on Firm Data. Industry and innovation, v. 18, n. 4, p. 369-392, 2011.

ASHEIM, B.T.; ISAKSEN, A. Regional innovation systems: the integration of local 'sticky' and global 'ubiquitous' knowledge. The Journal of Technology Transfer, v. 27, n. 1, p. 77-86, 2002.

BALESTRIN, A.; VERSCHOORE, J. R. Redes de cooperação empresarial: estratégias de gestão na nova economia. Porto Alegre: Bookman, 2008.

BALLOU, R. H.; GILBERT, S. M.; MUKHERJEE, A. New managerial challenges from supply chain opportunities. Industrial Marketing Management, v. 29, n. 1, p. 7-18, 2000.

BASANT, R. Knowledge flows and industrial clusters: An analytical review of literature. East-West Center Working Paper, v. 40, p. 1-77, 2002.

BENTON, L. La emergencia de los distritos industriales en España: reconversión industrial y divergencia de respuestas regionales. Los Distritos Industriales y Las Pequeñas Empresas, Madrid, p. 81-127, 1993.

BERBEGAL-MIRABENT, J.; LAFUENTE, E.; SOLÉ, F. The pursuit of knowledge transfer activities: An efficiency analysis of Spanish universities. Journal of Business Research, v. 66, n. 10, p. 2051-2059, 2013.

BITTI, P. R.; ZANNI, B. A. Comunicação como Processo Social. Lisboa: Editorial Estampa, 1993.

BROOKING, A. Intellectual capital. Reino Unido: Cengage Learning EMEA, 1996.

BROWN, S.L; EISENHARDT, K. M. The art of continuous change: Linking complexity theory and timepaced evolution in relentlessly shifting organizations. Administration Science Quarterly, v. 42, n. 1, p. 1-34, 1997.

BRUSCO, S. The idea of the Industrial District: Its genesis. Industrial districts and inter-firm cooperation in Italy, Geneva, p. 10-19, 1990.

CASTELLS, M. Materials for an exploratory theory of the network society. British Journal of Sociology, v. 51, n. 1, p. 5-24, jan./mar., 2000. 
CASTELLS, M.; CARDOSO, G. The network society: from knowledge to policy. Washington: John Hopkins, 2005.

CASTRO, L. Strategizing across boundaries: revisiting knowledge brokering activities in French innovation clusters. Journal of Knowledge Management, v. 19, n. 5, p. 1048-1068, 2015.

CHENG, H.; NIU, M.; NIU, K. Industrial cluster involvement, organizational learning, and organizational adaptation: an exploratory study in high technology industrial districts. Journal of Knowledge Management, v. 18, n. 5, p. 971-990, 2014.

CLARK, K. B.; FUJIMOTO, T. Product development performance: Strategy, organization, and management in the world auto industry. Harvard: Harvard Business Press, 1991.

CONNELL, J.; VOOLA, R. Knowledge integration and competitiveness: a longitudinal study of an industry cluster. Journal of Knowledge Management, v. 17, n. 2, p. 208-225, 2013.

CORREIA, I. Spillovers de Conhecimento e Desenvolvimento Regional: Evidência de Portugal. Revista portuguesa de estudos regionais, n. 13, p. 67-80, 2007.

COOKE, P. The co-operative advantage of regions. In: BARNES, T.; GERTLER, M. (Eds.). The new industrial geography: Regions, regulation, and institutions. London: Routledge, 1999. p. 54-73.

DARR, E.; ARGOTE, L.; EPPLE, D. The acquisition, transfer and depreciation of knowledge in service organizations: Productivity in franchises. Management Science, v. 41, p. 1750-1762, 1995.

DAVENPORT, T. H.; PRUSAK, L. Conhecimento empresarial. Rio de Janeiro: Campus, 1998.

EBERS, M.; JARILLO, J. C. The construction, forms, and consequences of industry networks. International Studies of Management and Organization, v. 27, n. 4, p. 3, 1998.

ETZKOWITZ, H. Innovation in innovation: the triple helix of university-industry-government relations, Social Science Information, v. 42, n. 3, p. 293-337, 2003.

FIGUEIREDO, J. C; DI SERIO, L. C. Estratégia em clusters empresariais: conceitos e impacto na competitividade. In: DI SERIO, L. C. (Org). Clusters empresariais no Brasil: casos selecionados. São Paulo: Saraiva, 2007. p. 1-18.

FOMBRUN, C.J. Strategies for Network Research in Organizations. The Academy of Management Review, v. 7, n. 2, p. 280-291, 1982.

GIL, A. C. Métodos e técnicas de pesquisa social. 6. ed. São Paulo: Saraiva, 2008.

GRANDORI, A.; SODA, G.; Inter-firm networks: Antecedents, mechanisms and forms. Organization Studies, v. 16, n. 2, p. 183-214, 1995.

GRANOVETTER, M. Economic Action and Social Structure: The problem of embeddedness. American Journal of sociology, v. 91, p. 481-510, 1985. 
GRIMPE, C.; HUSSINGER, K. Formal and Informal Knowledge and Technology Transfer from Academia to Industry: Complementarity Effects and Innovation Performance. Industry and Innovation, v. 20, n. 8, p. 683-700, 2013.

HOFFMANN, E.; LOPES, G.; MEDEIROS, J. Knowledge transfer among the small businesses of a Brazilian cluster. Journal of Business Research, v. 67, n 5, p. 856-864, 2014.

JARVENPAA, S. L.; WERNICK A. Paradoxical tensions in open innovation networks. European Journal of Innovation Management, v. 14, n. 4, p. 521-548, 2011.

KRAATZ, M. S. Learning by association? International networks and adaptation to environmental change. Academy of Management Journal, v. 41, n. 6, p. 621-643, 1998.

LASTRES, H.M.; CASSIOLATO, J. E. Glossário de arranjos e sistemas produtivos e inovativos locais. Rio de Janeiro: IE, 2003.

MALMBERG, A.; POWER, D. How do firms in clusters create knowledge? Uppsala University, v. 12, p- 409-431, 2005.

MARSHALL, A. Principles of Economics Book Four: The Agents of Production: Land, Labour, and Capital and Organization. [S.I.]:[s.n.], 1890. [Versão Eletrônica].

MASCENA, K. M. C.; FIGUEIREDO, F. C.; BOAVENTURA, J. M. G. Clusters e APL's: análise bibliométrica das publicações nacionais no período de 2000 a 2011. Revista de Administração de Empresas (RAE), v. 53, p. 454-468, 2013.

MILES, R., SNOW, C. Organizations: New concepts for new forms. California Management Review, n. 28, p. 62-73, 1986.

MINAYO, M. C. S.. O desafio do conhecimento: pesquisa qualitativa em saúde. 7. ed. São Paulo: Hucitec; Rio de Janeiro: Abrasco, 2000.

MINTZBERG, $H$. The structuring of organizations: $A$ synthesis of the research. [1979]. Disponível em: <https://papers.ssrn.com/sol3/papers.cfm?abstract_id=1496182>. Acesso em: 30 nov. 2016.

MOLINA-MORALES, F. X. Human capital in the industrial districts. Human Systems Management, v. 20, p. 319-331, 2001.

MOROSINI, P. Industrial clusters, knowledge integration and performance. World Development, v. 32, p. 305-326, 2004.

NOHRIA, N.; ECLES, R. Networks and organizations: Structure, form, and action. Boston: Harvard Business School, 1992.

NONAKA, I.; TAKEUCHI, H. Criação de conhecimento na empresa. Rio de Janeiro: Campus, 1997.

NOVELI, M.; SEGATTO, A. P. Processo de Cooperação Universidade Empresa para a Inovação Tecnológica em um Parque Tecnológico: evidências empíricas e proposição de um modelo conceitual. Revista de Administração e Inovação (RAI), v. 9, n. 1, p. 81-105, 2012. 
OLIVER, A. L.; EBERS, M. Networking network studies: an analysis of conceptual configurations in the study of inter-organizational relationships. Organization Studies, v. 19, p. 549-583, 1998.

PAULIN, D.; SUNESON, K. Knowledge transfer, knowledge sharing and knowledge barriers-three blurry terms in KM. Leading Issues in Knowledge Management, v. 2, p. 73, 2015.

PAULIN, D.; WINROTH, M. Facilitators, Inhibitors, and Obstacles-A Refined Categorization Regarding Barriers for Knowledge Transfer, Sharing, and Flow. In: International Conference on Intellectual Capital, Knowledge Management and Organizational Learning, 10., 2013, Washington, DC, USA. Proceedings... Washington, DC: Academic Conferences Ltd. p. 320-328.

POLANYI, M. The tacit dimension. Garden City, NY: Anchor, 1967.

PORTER, M. E. The competitive advantage of nations. New York: Free Press, 1990.

POWELL, W. Learning from collaboration: Knowledge and networks in the biotechnology and pharmaceutical industries. California Management Review, v. 40, p. 228-240, 1998.

POWELL, W; KOPUT, K.W.; SMITH-DOERR, L. Interorganizational collaboration and the locus of innovation: Networks of learning in biotechnology. Administrative science quarterly, v. 41, n. 11, 1996. p. 116-145.

RICHARDSON, R. J. Pesquisa social: métodos e técnicas. 3. ed. São Paulo: Atlas, 1999.

RUBIN, T. H.; AAS, T.H.; STEAD, A. Knowledge flow in technological business incubators: evidence from Australia and Israel. Technovation, v. 41, p. 11-24, 2015.

SARACH, L. Analysis of Cooperative Relationship in Industrial Cluster. Procedia - Social and Behavioral Sciences, v. 191, p. 250-254, 2015.

SAWHNEY, M; PARIKH, D. Where value lives in a network world. Harvard Bussiness Review, Boston, v. 71, n. 1, p. 79-92, 2001.

SIEGEL, D. S.; WALDMAN, D.; LINK, A. Assessing the impact of organizational practices on the relative productivity of university technology transfer offices: an exploratory study. Research policy, v. 32, n. 1, p. 27-48, 2003.

SINGLEY, M. K., ANDERSON, J. R. The transfer of cognitive skill. Cambridge: Harvard University Press, 1989.

SOHN, A. et al. Aprendizagem interorganizacional: estudo sobre os canais de transmissão de conhecimento em cluster têxteis e de vestuário no Brasil e em Portugal. Revista Alcance, v. 21, n. 4, p. 777-796, 2014.

STAIR, R. M.; REYNOLDS G.W. (Colab.) Princípios de sistemas de informação: uma abordagem gerencial. Trad. Alexandre Melo de Oliveira. Rio de Janeiro: LTC, 2002.

SVEIBY, K. E. Methods for measuring intangible assets. [2001]. Disponível em: <http://www.sveiby.com/articles/IntangibleMethods.htm >. Acesso em 23 jan. 2016. 
SZULANSKI, G. Exploring internal stickiness: impediments to transfer of best practice within the firm. Strategic Management Journal, v. 17, p. 27-43, 1996.

SZULANSKI, G. The process of knowledge transfer: A diachronic analysis of stickiness. Organizational behavior and human decision processes, v. 82, n. 1, p. 9-27, 2000.

TALLMAN, S. et al. Knowledge, clusters, and competitive advantage. Academy of management review, v. 29, n. 2, p. 258-271, 2004.

TODEVA, E. Clusters in the South East of England. University of Surrey. 2006. Disponível em: $<$ https://www.surrey.ac.uk/sbs/sar/centres/bcned/BCNED\%20Files/SEEDA\%20Cluster\%20Report.p df $>$. Acesso em: 24 jan. 2016.

TODEVA, E.; KNOKE, D.; KESKINOVA, D. Porous and Fuzzy Boundaries. Minnesota: University of Minnesota, 2007.

TZORTZAKI, A. M.; MIHIOTIS, A. A review of knowledge management theory and future directions. Knowledge and Process Management, v. 21, n. 1, p. 29-41, 2014.

UZZI, B. Social structure and competition in interfirm networks: The paradox of embeddedness. Administrative Science Quarterly, v. 42, n. 1, p. 35-67, 1997.

VÁSQUEZ-URRIAGO, A.R.; BARGE-GIL, A.; RICO, M. Science and Technology Parks and cooperation for innovation: Empirical evidence from Spain, Research Policy, v. 45, p. 137-147, 2016.

WILLIAMSON, O. E. Transaction-cost economics: the governance of contractual relations. Journal of law and economics, v. 22, n. 02, p. 233-261, 1979.

ZACCARELLI, S. B. et al. Clusters e Redes de Negócios: uma nova visão para a gestão dos negócios. São Paulo: Atlas, 2008.

ZELENY, M. Human systems management: Integrating knowledge, management and systems. Singapore: World Scientific, 2005.

ZENG, D. Z. et al. (Ed.). Knowledge, technology, and cluster-based growth in Africa. [2008]. Disponível

em:<https://www.innovationpolicyplatform.org/sites/default/files/rdf_imported_documents/kno wledge_cluster_africa_2008.pdf >. Acesso em: 14 jan. 2016. 